\title{
GENERALIZED FOURIER-FEYNMAN TRANSFORM AND SEQUENTIAL TRANSFORMS ON FUNCTION SPACE
}

\author{
Jae Gil Choi and Seung Jun Chang*
}

\begin{abstract}
In this paper we first investigate the existence of the generalized Fourier-Feynman transform of the functional $F$ given by

$$
F(x)=\hat{\nu}\left(\left(e_{1}, x\right)^{\sim}, \ldots,\left(e_{n}, x\right)^{\sim}\right),
$$

where $(e, x)^{\sim}$ denotes the Paley-Wiener-Zygmund stochastic integral with $x$ in a very general function space $C_{a, b}[0, T]$ and $\hat{\nu}$ is the Fourier transform of complex measure $\nu$ on $\mathcal{B}\left(\mathbb{R}^{n}\right)$ with finite total variation. We then define two sequential transforms. Finally, we establish that the one is to identify the generalized Fourier-Feynman transform and the another transform acts like an inverse generalized Fourier-Feynman transform.
\end{abstract}

\section{Introduction}

Let $C_{0}[0, T]$ denote one-parameter Wiener space. The concept of an $L_{1}$ analytic Fourier-Feynman transform(FFT) for functionals on Wiener space $C_{0}[0, T]$ was introduced by Brue in [1]. This transform and its properties are similar in many respects to the ordinary Fourier function transform. Further works involving the $L_{2}-L_{2}$ theory and the $L_{p}-L_{p^{\prime}}$ theory, $1 / p+1 / p^{\prime}=1$, includes $[2,14]$. In [10], Huffman, Park and Skoug defined a convolution product for functionals on Wiener space, and they obtained various results for the analytic FFT and the convolution product [10], [11], [12]. Also see the references in [16] for further informations and results.

In [9], Chang and Skoug defined a generalized analytic Fourier-Feynman transform (GFFT) for functionals on very general function space $C_{a, b}[0, T]$. The function space $C_{a, b}[0, T]$, induced by generalized Brownian motion process, was introduced by J. Yeh $[17,18]$ and was used extensively by Chang and Chung [7], Chang and Skoug [9], and Chang, Chung and Skoug [8]. The GFFT theory on $C_{a, b}[0, T]$ have been developed in many papers including $[3,5,6,9]$.

Received July 29, 2011.

2010 Mathematics Subject Classification. 28C20, 60J65.

Key words and phrases. generalized Brownian motion process, Paley-Wiener-Zygmund stochastic integral, cylinder functional, generalized Fourier-Feynman transform, sequential $\mathcal{P}$-transform, sequential $\mathcal{N}$-transform.

* The present research was conducted by the research fund of Dankook University in 2009. 
In this paper we investigate the existence of the GFFT for bounded cylinder functionals of the form

$$
F(x)=\hat{\nu}\left(\left(e_{1}, x\right)^{\sim}, \ldots,\left(e_{n}, x\right)^{\sim}\right),
$$

where $(e, x)^{\sim}$ denotes the Paley-Wiener-Zygmund stochastic integral with $x$ in function space $C_{a, b}[0, T]$ and $\hat{\nu}$ is the Fourier transform of complex measure $\nu$ on $\mathcal{B}\left(\mathbb{R}^{n}\right)$ with finite total variation. We define sequential $\mathcal{P}$ and $\mathcal{N}$ function space transforms of such cylinder functionals. We then study relationships between the sequential $\mathcal{P}$-transform and the GFFT of the functionals on $C_{a, b}[0, T]$ and present a version of the inverse GFFT via the sequential $\mathcal{N}$-transform.

The Wiener process used in [1], [2], [10], [11], [12], [14] is free of drift and stationary in time while the stochastic process used in this paper, as well as in $[3],[4],[5],[6],[7],[8],[9]$ is nonstationary in time and is subject to a drift $a(t)$. It turns out, as noted in Remark 4.1 below, that including a drift term $a(t)$ makes establishing the existence of the GFFT of functionals on $C_{a, b}[0, T]$ very difficult. However, when $a(t) \equiv 0$ and $b(t)=t$ on $[0, T]$, the general function space $C_{a, b}[0, T]$ reduces to the Wiener space $C_{0}[0, T]$.

\section{Preliminaries}

Let $D=[0, T]$ and let $(\Omega, \mathcal{W}, P)$ be a probability measure space. A realvalued stochastic process $Y$ on $(\Omega, \mathcal{W}, P)$ and $D$ is called a generalized Brownian motion process if $Y(0, \omega)=0$ almost everywhere and for $0=t_{1}<t_{2}<\cdots<$ $t_{n} \leq T$, the $n$-dimensional random vector $\left(Y\left(t_{1}, \omega\right), \ldots, Y\left(t_{n}, \omega\right)\right)$ is normally distributed with density function

$$
\begin{aligned}
K_{n}(\vec{t}, \vec{u})= & \left(\prod_{j=1}^{n} 2 \pi\left(b\left(t_{j}\right)-b\left(t_{j-1}\right)\right)\right)^{-1 / 2} \\
& \times \exp \left\{-\frac{1}{2} \sum_{j=1}^{n} \frac{\left[\left(u_{j}-a\left(t_{j}\right)\right)-\left(u_{j-1}-a\left(t_{j-1}\right)\right)\right]^{2}}{b\left(t_{j}\right)-b\left(t_{j-1}\right)}\right\},
\end{aligned}
$$

where $\vec{u}=\left(u_{1}, \ldots, u_{n}\right), u_{0}=0, \vec{t}=\left(t_{1}, \ldots, t_{n}\right), a(t)$ is an absolutely continuous real-valued function on $[0, T]$ with $a(0)=0, a^{\prime}(t) \in L^{2}[0, T]$, and $b(t)$ is a strictly increasing, continuously differentiable real-valued function with $b(0)=$ 0 and $b^{\prime}(t)>0$ for each $t \in[0, T]$.

In [18], Yeh shows that the generalized Brownian motion process $Y$ determined by $a(\cdot)$ and $b(\cdot)$ is a Gaussian process with mean function $a(t)$ and covariance function $r(s, t)=\min \{b(s), b(t)\}$, and that the probability measure $\mu$ induced by $Y$, taking a separable version, is supported by $C_{a, b}[0, T]$ (which is equivalent to the Banach space of continuous functions $x$ on $[0, T]$ with $x(0)=0$ under the sup norm). Hence, $\left(C_{a, b}[0, T], \mathcal{B}\left(C_{a, b}[0, T]\right), \mu\right)$ is the function space induced by $Y$ where $\mathcal{B}\left(C_{a, b}[0, T]\right)$ is the Borel $\sigma$-algebra of $C_{a, b}[0, T]$. We then complete this function space to obtain $\left(C_{a, b}[0, T], \mathcal{W}\left(C_{a, b}[0, T]\right), \mu\right)$ where $\mathcal{W}\left(C_{a, b}[0, T]\right)$ is the set of all Wiener measurable subsets of $C_{a, b}[0, T]$. 
A subset $B$ of $C_{a, b}[0, T]$ is said to be scale-invariant measurable [13] provided $\rho B \in \mathcal{W}\left(C_{a, b}[0, T]\right)$ for all $\rho>0$, and a scale-invariant measurable set $N$ is said to be scale-invariant null provided $\mu(\rho N)=0$ for all $\rho>0$. A property that holds except on a scale-invariant null set is said to be hold scale-invariant almost everywhere(s-a.e.). If two functionals $F$ and $G$ are equal s-a.e., we write $F \approx G$.

In this paper, as in [3], [4], [5], [6], [8], [9], we consider the incomplete function space $\left(C_{a, b}[0, T], \mathcal{B}\left(C_{a, b}[0, T]\right), \mu\right)$ and we denote the function space integral of a $\mathcal{B}\left(C_{a, b}[0, T]\right)$-measurable functional $F$ by

$$
E[F]=\int_{C_{a, b}[0, T]} F(x) d \mu(x)
$$

whenever the integral exists.

Let $L_{a, b}^{2}[0, T]$ be the separable Hilbert space of functions on $[0, T]$ which are Lebesgue measurable and square integrable with respect to the LebesgueStieltjes measures on $[0, T]$ induced by $b(t)$ and $a(t)$ : i.e.,

$$
L_{a, b}^{2}[0, T]=\left\{v: \int_{0}^{T} v^{2}(s) d b(s)<+\infty \text { and } \int_{0}^{T} v^{2}(s) d|a|(s)<+\infty\right\},
$$

where $|a|(t)$ denote the total variation function of $a(t)$. The inner product on $L_{a, b}^{2}[0, T]$ is defined by $(u, v)_{a, b}=\int_{0}^{T} u(t) v(t) d[b(t)+|a|(t)]$. Note that $\|u\|_{a, b}=\sqrt{(u, u)_{a, b}}=0$ if and only if $u(t)=0$ a.e. on $[0, T]$ and that all functions of bounded variation on $[0, T]$ are elements of $L_{a, b}^{2}[0, T]$. Also note that if $a(t) \equiv 0$ and $b(t)=t$, then $L_{a, b}^{2}[0, T]=L^{2}[0, T]$. In fact,

$$
\left(L_{a, b}^{2}[0, T],\|\cdot\|_{a, b}\right) \subset\left(L_{0, b}^{2}[0, T],\|\cdot\|_{0, b}\right)=\left(L^{2}[0, T],\|\cdot\|_{2}\right)
$$

since the two norms $\|\cdot\|_{0, b}$ and $\|\cdot\|_{2}$ are equivalent.

For each $v \in L_{a, b}^{2}[0, T]$, the Paley-Wiener-Zygmund(PWZ) stochastic integral $\langle v, x\rangle$ is given by the formula

$$
\langle v, x\rangle=\lim _{n \rightarrow \infty} \int_{0}^{T} \sum_{j=1}^{n}\left(v, \phi_{j}\right)_{a, b} \phi_{j}(t) d x(t)
$$

for $\mu$-a.e. $x \in C_{a, b}[0, T]$, where $\left\{\phi_{j}\right\}_{j=1}^{\infty}$ is a complete orthonormal set of real-valued functions of bounded variation on $[0, T]$ such that $\left(\phi_{j}, \phi_{k}\right)_{a, b}=\delta_{j k}$ (the Kronecker delta). If $v$ is of bounded variation on $[0, T]$, then the PWZ stochastic integral $\langle v, x\rangle$ equals the Riemann-Stieltjes integral $\int_{0}^{T} v(t) d x(t)$ for s-a.e. $x \in C_{a, b}[0, T]$.

Let

$$
C_{a, b}^{\prime}[0, T]=\left\{w \in C_{a, b}[0, T]: w(t)=\int_{0}^{t} z(s) d b(s) \text { for some } z \in L_{a, b}^{2}[0, T]\right\} .
$$


For $w \in C_{a, b}^{\prime}[0, T]$, with $w(t)=\int_{0}^{t} z(s) d b(s)$ for $t \in[0, T]$, let $D_{t}: C_{a, b}^{\prime}[0, T] \rightarrow$ $L_{a, b}^{2}[0, T]$ be defined by the formula

$$
D_{t} w=z(t)=\frac{w^{\prime}(t)}{b^{\prime}(t)} .
$$

Then $C_{a, b}^{\prime} \equiv C_{a, b}^{\prime}[0, T]$ with inner product

$$
\left(w_{1}, w_{2}\right)_{C_{a, b}^{\prime}}=\int_{0}^{T} D_{t} w_{1} D_{t} w_{2} d b(t)=\int_{0}^{T} z_{1}(t) z_{2}(t) d b(t)
$$

is a separable Hilbert space. Note that the two separable Hilbert spaces $L_{a, b}^{2}[0, T]$ and $C_{a, b}^{\prime}[0, T]$ are homeomorphic.

Throughout this paper we assume $a \in C_{a, b}^{\prime}[0, T]$ and, for notational convenience, we use the notation $(w, x)^{\sim}$ instead of $\left\langle D_{t} w, x\right\rangle$. Then we see that for each $w$ in $C_{a, b}^{\prime}[0, T]$, the random variable $x \mapsto(w, x)^{\sim}$ is Gaussian with mean $(w, a)_{C_{a, b}^{\prime}}$ and variance $\|w\|_{C_{a, b}^{\prime}}^{2}$, and that $(w, \alpha x)^{\sim}=\alpha(w, x)^{\sim}=(\alpha w, x)^{\sim}$ for any real number $\alpha, w \in C_{a, b}^{\prime}[0, T]$ and $x \in C_{a, b}[0, T]$. We also see that if $\left\{w_{1}, \ldots, w_{n}\right\}$ is an orthonormal set in $C_{a, b}^{\prime}[0, T]$, then the random variables $\left(w_{i}, x\right)^{\sim}$ 's are independent. From these we obtain the following integration formula for the function space $C_{a, b}[0, T]$.

Let $\left\{e_{1}, \ldots, e_{n}\right\}$ be an orthonormal set in $C_{a, b}^{\prime}[0, T]$, let $g: \mathbb{R}^{n} \rightarrow \mathbb{C}$ be a Borel measurable function and let $G: C_{a, b}[0, T] \rightarrow \mathbb{C}$ be given by equation

$$
G(x)=g\left(\left(e_{1}, x\right)^{\sim}, \ldots,\left(e_{n}, x\right)^{\sim}\right) .
$$

Then

$$
\begin{aligned}
E[G]= & \int_{C_{a, b}[0, T]} g\left(\left(e_{1}, x\right)^{\sim}, \ldots,\left(e_{n}, x\right)^{\sim}\right) d \mu(x) \\
= & (2 \pi)^{-n / 2} \int_{\mathbb{R}^{n}} g\left(u_{1}, \ldots, u_{n}\right) \\
& \quad \times \exp \left\{-\sum_{j=1}^{n} \frac{\left[u_{j}-\left(e_{j}, a\right)_{C_{a, b}^{\prime}}\right]^{2}}{2}\right\} d u_{1} \cdots d u_{n}
\end{aligned}
$$

in the sense that if either side of equation (2.1) exists, both sides exist and equality holds.

Throughout this paper, let $\mathbb{C}, \mathbb{C}_{+}$and $\tilde{\mathbb{C}}_{+}$denote the complex numbers, the complex numbers with positive real part, and the nonzero complex numbers with nonnegative real part, respectively. For each $\lambda \in \tilde{\mathbb{C}}_{+}, \lambda^{-1 / 2}\left(\right.$ or $\left.\lambda^{1 / 2}\right)$ is always chosen to have positive real part. The following integration formula is used several times in this paper:

$$
\int_{\mathbb{R}} \exp \left\{-\alpha u^{2}+\beta u\right\} d u=\left(\frac{\pi}{\alpha}\right)^{1 / 2} \exp \left\{\frac{\beta^{2}}{4 \alpha}\right\}
$$

for complex numbers $\alpha$ and $\beta$ with $\operatorname{Re}(\alpha)>0$. 


\section{Bounded cylinder functionals}

In this section, we introduce a class of certain bounded cylinder functionals and state the concepts of the generalized analytic Feynman integral and the GFFT on function space $C_{a, b}[0, T]$. We then establish the existence of the GFFT of such cylinder functionals.

A functional $F$ is called a cylinder functional on $C_{a, b}[0, T]$ if there exists a finite subset $\left\{h_{1}, \ldots, h_{m}\right\}$ of $C_{a, b}^{\prime}[0, T]$ such that

$$
F(x)=\phi\left(\left(h_{1}, x\right)^{\sim}, \ldots,\left(h_{m}, x\right)^{\sim}\right), \quad x \in C_{a, b}[0, T],
$$

where $\phi$ is a complex-valued Borel measurable function on $\mathbb{R}^{m}$. It is easy to show that for given cylinder functional $F$ of the form (3.1) there exists an orthonormal subset $\left\{e_{1}, \ldots, e_{n}\right\}$ of $C_{a, b}^{\prime}[0, T]$ such that $F$ is expressed as

$$
F(x)=f\left(\left(e_{1}, x\right)^{\sim}, \ldots,\left(e_{n}, x\right)^{\sim}\right), \quad x \in C_{a, b}[0, T],
$$

where $f$ is a complex-valued Borel measurable function on $\mathbb{R}^{n}$. Thus we lose no generality in assuming that every cylinder functional on $C_{a, b}[0, T]$ is of the form (3.2).

Definition 3.1. Let $\mathcal{M}\left(\mathbb{R}^{n}\right)$ denote the space of complex-valued Borel measures on $\mathcal{B}\left(\mathbb{R}^{n}\right)$. It is well known that a complex-valued Borel measure $\nu$ necessarily has a finite total variation $\|\nu\|$, and $\mathcal{M}\left(\mathbb{R}^{n}\right)$ is a Banach algebra under the norm $\|\cdot\|$ and with convolution as multiplication.

For $\nu \in \mathcal{M}\left(\mathbb{R}^{n}\right)$, the Fourier transform $\hat{\nu}$ of $\nu$ is a complex-valued function defined on $\mathbb{R}^{n}$ by the formula

$$
\hat{\nu}(\vec{u})=\int_{\mathbb{R}^{n}} \exp \left\{i \sum_{j=1}^{n} u_{j} v_{j}\right\} d \nu(\vec{v}),
$$

where $\vec{u}=\left(u_{1}, \ldots, u_{n}\right)$ and $\vec{v}=\left(v_{1}, \ldots, v_{n}\right)$ are in $\mathbb{R}^{n}$.

Let $\left\{e_{1}, \ldots, e_{n}\right\}$ be an orthonormal subset of $C_{a, b}^{\prime}[0, T]$. Define the functional $F: C_{a, b}[0, T] \rightarrow \mathbb{C}$ by

$$
F(x)=\hat{\nu}\left(\left(e_{1}, x\right)^{\sim}, \ldots,\left(e_{n}, x\right)^{\sim}\right), \quad x \in C_{a, b}[0, T],
$$

where $\hat{\nu}$ is the Fourier transform of $\nu$ in $\mathcal{M}\left(\mathbb{R}^{n}\right)$. Then $F$ is a bounded cylinder functional and $|\hat{\nu}(\vec{u})| \leq\|\nu\|<+\infty$. Throughout this paper, we fix the positive integer $n$.

Next (see $[9,5,6,4]$ ) we state the definition of the generalized analytic Feynman integral and the GFFT.

Definition 3.2. Let $F: C_{a, b}[0, T] \rightarrow \mathbb{C}$ be a scale-invariant measurable functional such that the function space integral $J(\lambda)=E\left[F\left(\lambda^{-1 / 2} \cdot\right)\right]$ exists as a finite number for all $\lambda>0$. If there exists a function $J^{*}(\lambda)$ analytic in $\mathbb{C}_{+}$ such that $J^{*}(\lambda)=J(\lambda)$ for all $\lambda>0$, then $J^{*}(\lambda)$ is defined to be the analytic 
function space integral of $F$ over $C_{a, b}[0, T]$ with parameter $\lambda$, and for $\lambda \in \mathbb{C}_{+}$ we write

$$
E^{\operatorname{an}_{\lambda}}[F] \equiv E_{x}^{\mathrm{an}_{\lambda}}[F(x)]=J^{*}(\lambda) .
$$

Let $q \neq 0$ be a real number and let $F$ be a functional such that $E^{\operatorname{an}_{\lambda}}[F]$ exists for all $\lambda \in \mathbb{C}_{+}$. If the following limit exists, we call it the generalized analytic Feynman integral of $F$ with parameter $q$ and we write

$$
E^{\operatorname{anf}_{q}}[F] \equiv E_{x}^{\operatorname{anf}_{q}}[F(x)]=\lim _{\lambda \rightarrow-i q} E^{\operatorname{an}_{\lambda}}[F],
$$

where $\lambda \rightarrow-i q$ through values in $\mathbb{C}_{+}$.

Definition 3.3. For $\lambda \in \mathbb{C}_{+}$and $y \in C_{a, b}[0, T]$, let

$$
T_{\lambda}(F)(y)=E_{x}^{\mathrm{an}_{\lambda}}[F(y+x)] .
$$

For $p \in(1,2]$, we define the $L_{p}$ analytic $\operatorname{GFFT}, T_{q}^{(p)}(F)$ of $F$, by the formula $\left(\lambda \in \mathbb{C}_{+}\right)$,

$$
T_{q}^{(p)}(F)(y)=\underset{\lambda \rightarrow-i q}{\lim . \operatorname{m}} T_{\lambda}(F)(y)
$$

if it exists; i.e., for each $\rho>0$,

$$
\lim _{\lambda \rightarrow-i q} \int_{C_{a, b}[0, T]}\left|T_{\lambda}(F)(\rho y)-T_{q}^{(p)}(F)(\rho y)\right|^{p^{\prime}} d \mu(y)=0,
$$

where $1 / p+1 / p^{\prime}=1$. We define the $L_{1}$ analytic $\operatorname{GFFT}, T_{q}^{(1)}(F)$ of $F$, by the formula $\left(\lambda \in \mathbb{C}_{+}\right)$

$$
T_{q}^{(1)}(F)(y)=\lim _{\lambda \rightarrow-i q} T_{\lambda}(F)(y)
$$

if it exists.

We note that for $1 \leq p \leq 2, T_{q}^{(p)}(F)$ is defined only s-a.e.. We also note that if $T_{q}^{(p)}(F)$ exists and if $F \approx G$, then $T_{q}^{(p)}(G)$ exists and $T_{q}^{(p)}(G) \approx T_{q}^{(p)}(F)$. From the definition of the $L_{1}$ analytic GFFT, we can see that for $q \in \mathbb{R}-\{0\}$,

$$
E^{\operatorname{anf}_{q}}[F]=T_{q}^{(1)}(F)(0)
$$

if it exists [5, 9].

Lemma 3.4. Let $\left\{e_{1}, \ldots, e_{n}\right\}$ be an orthonormal subset of $C_{a, b}[0, T]$. Then for all $\zeta \in \mathbb{C}_{+}$, the function space integral

$$
E_{x}\left[\exp \left\{i \zeta \sum_{j=1}^{n}\left(e_{j}, x\right)^{\sim} v_{j}\right\}\right]
$$

exists and is given by the formula

$$
E_{x}\left[\exp \left\{i \zeta \sum_{j=1}^{n}\left(e_{j}, x\right)^{\sim} v_{j}\right\}\right]=\exp \left\{-\frac{\zeta^{2}}{2} \sum_{j=1}^{n} v_{j}^{2}+i \zeta \sum_{j=1}^{n}\left(e_{j}, a\right)_{C_{a, b}^{\prime}} v_{j}\right\} .
$$

Proof. Using (2.1), the Fubini theorem, and (2.2), it follows that the equation (3.7) holds for all $\zeta \in \mathbb{C}_{+}$. 
For convenience we use the following notation throughout this paper:

$$
\begin{aligned}
\psi_{\vec{e}}(\lambda ; \vec{v}) & \equiv \psi_{e_{1}, \ldots, e_{n}}\left(\lambda ; v_{1}, \ldots, v_{n}\right) \\
& =\exp \left\{-\frac{1}{2 \lambda} \sum_{j=1}^{n} v_{j}^{2}+i \lambda^{-1 / 2} \sum_{j=1}^{n}\left(e_{j}, a\right)_{C_{a, b}^{\prime}} v_{j}\right\}
\end{aligned}
$$

for an orthonormal subset $\left\{e_{1}, \ldots, e_{n}\right\}$ of $C_{a, b}^{\prime}[0, T], \lambda \in \tilde{\mathbb{C}}_{+}$and $\vec{v}=\left(v_{1}, \ldots\right.$, $\left.v_{n}\right) \in \mathbb{R}^{n}$.

In next theorem, we show that the analytic function space integral $T_{\lambda}(F)(y)$ $=E_{x}^{a n_{\lambda}}[F(y+x)]$ of the functional $F$ given by equation (3.4) exists.

Theorem 3.5. Let $F$ be given by the equation (3.4). Then for all $\lambda \in \mathbb{C}_{+}$, $T_{\lambda}(F)$ exists and is given by the formula

$$
T_{\lambda}(F)(y)=\int_{\mathbb{R}^{n}} \exp \left\{i \sum_{j=1}^{n}\left(e_{j}, y\right)^{\sim} v_{j}\right\} \psi_{\vec{e}}(\lambda ; \vec{v}) d \nu(\vec{v}),
$$

where $\psi_{\vec{e}}(\lambda ; \vec{v})$ is given by the equation (3.8).

Proof. By (3.4), (3.3), the Fubini theorem, (3.7) with $\zeta$ replaced with $\lambda^{-1 / 2}$, and (3.8), we have that for all $\lambda>0$,

$$
J(\lambda)=E_{x}\left[F\left(y+\lambda^{-1 / 2} x\right)\right]=\int_{\mathbb{R}^{n}} \exp \left\{i \sum_{j=1}^{n}\left(e_{j}, y\right)^{\sim} v_{j}\right\} \psi_{\vec{e}}(\lambda ; \vec{v}) d \nu(\vec{v}) .
$$

Now let

$$
J^{*}(\lambda)=\int_{\mathbb{R}^{n}} \exp \left\{i \sum_{j=1}^{n}\left(e_{j}, y\right)^{\sim} v_{j}\right\} \psi_{\vec{e}}(\lambda ; \vec{v}) d \nu(\vec{v})
$$

for $\lambda \in \mathbb{C}_{+}$. Then $J^{*}(\lambda)=J(\lambda)$ for all $\lambda>0$. We will use the Morera theorem to show that $J^{*}(\lambda)$ is analytic on $\mathbb{C}_{+}$. Let $\left\{\lambda_{l}\right\}_{l=1}^{\infty}$ be a sequence in $\mathbb{C}_{+}$such that $\lambda_{l} \rightarrow \lambda$ through $\mathbb{C}_{+}$. Then $\lambda_{l}^{-1 / 2} \rightarrow \lambda^{-1 / 2}$ and $\operatorname{Re}\left(\lambda_{l}\right) \neq 0$ for all $l \in \mathbb{N}$. Thus we have that for each $l \in \mathbb{N}$,

$$
\begin{aligned}
&\left|\exp \left\{i \sum_{j=1}^{n}\left(e_{j}, y\right)^{\sim} v_{j}\right\} \psi_{\vec{e}}\left(\lambda_{l} ; \vec{v}\right)\right|=\left|\psi_{\vec{e}}\left(\lambda_{l} ; \vec{v}\right)\right| \\
&=\left|\exp \left\{-\frac{1}{2 \lambda_{l}} \sum_{j=1}^{n} v_{j}^{2}+i \lambda_{l}^{-1 / 2} \sum_{j=1}^{n}\left(e_{j}, a\right)_{C_{a, b}^{\prime}} v_{j}\right\}\right| \\
&= \exp \left\{-\frac{1}{2} \sum_{j=1}^{n}\left(\frac{\sqrt{\operatorname{Re}\left(\lambda_{l}\right)}}{\left|\lambda_{l}\right|} v_{j}+\frac{\left|\lambda_{l}\right| \operatorname{Im}\left(\lambda_{l}^{-1 / 2}\right)}{\sqrt{\operatorname{Re}\left(\lambda_{l}\right)}}\left(e_{j}, a\right)_{C_{a, b}^{\prime}}\right)^{2}\right. \\
&\left.+\frac{1}{2} \sum_{j=1}^{n} \frac{\left|\lambda_{l}\right|^{2}\left(\operatorname{Im}\left(\lambda_{l}^{-1 / 2}\right)\right)^{2}}{\operatorname{Re}\left(\lambda_{l}\right)}\left(e_{j}, a\right)_{C_{a, b}^{\prime}}^{2}\right\}
\end{aligned}
$$




$$
\leq \exp \left\{\frac{\left|\lambda_{l}\right|^{2}\left(\operatorname{Im}\left(\lambda_{l}^{-1 / 2}\right)\right)^{2}}{2 \operatorname{Re}\left(\lambda_{l}\right)} \sum_{j=1}^{n}\left(e_{j}, a\right)_{C_{a, b}^{\prime}}^{2}\right\} .
$$

Since $\nu \in \mathcal{M}\left(\mathbb{R}^{n}\right)$, we see that

$$
\begin{aligned}
& \left|\int_{\mathbb{R}^{n}} \exp \left\{\frac{\left|\lambda_{l}\right|^{2}\left(\operatorname{Im}\left(\lambda_{l}^{-1 / 2}\right)\right)^{2}}{2 \operatorname{Re}\left(\lambda_{l}\right)} \sum_{j=1}^{n}\left(e_{j}, a\right)_{C_{a, b}^{\prime}}^{2}\right\} d \nu(\vec{v})\right| \\
\leq & \int_{\mathbb{R}^{n}} \exp \left\{\frac{\left|\lambda_{l}\right|^{2}\left(\operatorname{Im}\left(\lambda_{l}^{-1 / 2}\right)\right)^{2}}{2 \operatorname{Re}\left(\lambda_{l}\right)} \sum_{j=1}^{n}\left(e_{j}, a\right)_{C_{a, b}^{\prime}}^{2}\right\} d|\nu|(\vec{v}) \\
= & \exp \left\{\frac{\left|\lambda_{l}\right|^{2}\left(\operatorname{Im}\left(\lambda_{l}^{-1 / 2}\right)\right)^{2}}{2 \operatorname{Re}\left(\lambda_{l}\right)} \sum_{j=1}^{n}\left(e_{j}, a\right)_{C_{a, b}^{\prime}}^{2}\right\}\|\nu\|<+\infty
\end{aligned}
$$

for each $l \in \mathbb{N}$. Furthermore we have that

$$
\begin{aligned}
& \lim _{l \rightarrow \infty} \int_{\mathbb{R}^{n}} \exp \left\{\frac{\left|\lambda_{l}\right|^{2}\left(\operatorname{Im}\left(\lambda_{l}^{-1 / 2}\right)\right)^{2}}{2 \operatorname{Re}\left(\lambda_{l}\right)} \sum_{j=1}^{n}\left(e_{j}, a\right)_{C_{a, b}^{\prime}}^{2}\right\} d|\nu|(\vec{v}) \\
= & \int_{\mathbb{R}^{n}} \exp \left\{\frac{|\lambda|^{2}\left(\operatorname{Im}\left(\lambda^{-1 / 2}\right)\right)^{2}}{2 \operatorname{Re}(\lambda)} \sum_{j=1}^{n}\left(e_{j}, a\right)_{C_{a, b}^{\prime}}^{2}\right\} d|\nu|(\vec{v}) .
\end{aligned}
$$

Thus, by Theorem 4.17 in [15, p. 92], $J^{*}(\lambda)$ is continuous on $\mathbb{C}_{+}$. Since $k(\lambda) \equiv$ $\exp \left\{i \sum_{j=1}^{n}\left(e_{j}, y\right)^{\sim} v_{j}\right\} \psi_{\vec{e}}(\lambda ; \vec{v})$ is analytic on $\mathbb{C}_{+}$, applying the Fubini theorem, we have

$$
\int_{\triangle} J^{*}(\lambda) d \lambda=\int_{\mathbb{R}^{n}} \int_{\triangle} k(\lambda) d \lambda d \nu(\vec{v})=0
$$

for all rectifiable simple closed curve $\triangle$ lying in $\mathbb{C}_{+}$. Thus by the Morera theorem, $J^{*}(\lambda)$ is analytic on $\mathbb{C}_{+}$. Therefore the analytic function space integral $J^{*}(\lambda)=E_{x}^{\operatorname{an}_{\lambda}}[F(y+x)] \equiv T_{\lambda}(F)(y)$ exists and is given by equation (3.9).

\section{Generalized Fourier-Feynman transform}

The remark below will be very useful in the development of our results for the GFFT of functionals given by equation (3.4).

Remark 4.1. If $a(t) \equiv 0$ on $[0, T]$, then for all $F$ given by equation (3.4), the $L_{1}$ analytic GFFT $T_{q}^{(1)}(F)$ will always exist for all real $q \neq 0$ and be given by the formula

$$
\begin{aligned}
T_{q}^{(1)}(F)(y) & =\int_{\mathbb{R}^{n}} \exp \left\{i \sum_{j=1}^{n}\left(e_{j}, y\right)^{\sim} v_{j}\right\} \psi_{\vec{e}}(-i q ; \vec{v}) d \nu(\vec{v}) \\
& =\int_{\mathbb{R}^{n}} \exp \left\{i \sum_{j=1}^{n}\left(e_{j}, y\right)^{\sim} v_{j}-\frac{i}{2 q} \sum_{j=1}^{n} v_{j}^{2}\right\} d \nu(\vec{v}) .
\end{aligned}
$$

However for $a(t)$ as in Section 2, and proceeding formally using equations (3.4) and (3.9), we see that $T_{q}^{(1)}(F)(y)$ will be given by equation (4.2) below if it 
exists. But the integral on the right-hand side of (4.2) might not exist if the real part of

$$
\ln \psi_{\vec{e}}(-i q ; \vec{v})=\left\{-\frac{i}{2 q} \sum_{j=1}^{n} v_{j}^{2}+i(-i q)^{-1 / 2} \sum_{j=1}^{n}\left(e_{j}, a\right)_{C_{a, b}^{\prime}} v_{j}\right\}
$$

is positive. However, by Cauchy-Schwartz inequality,

$$
\left|\psi_{\vec{e}}(-i q ; \vec{v})\right| \leq \exp \left\{\frac{\|a\|_{C_{a, b}^{\prime}}}{\sqrt{|2 q|}} \sum_{j=1}^{n}\left|v_{j}\right|\right\}
$$

and so the $L_{1}$ GFFT $T_{q}^{(1)}(F)$ of $F$ will certainly exist provided the associated measure $\nu$ of $F$ satisfies the condition

$$
\int_{\mathbb{R}^{n}} \exp \left\{\frac{\|a\|_{C_{a, b}^{\prime}}}{\sqrt{|2 q|}} \sum_{j=1}^{n}\left|v_{j}\right|\right\} d|\nu|(\vec{v})<+\infty .
$$

Note that in case $a(t) \equiv 0$ and $b(t)=t$ on $[0, T]$, the function space $C_{a, b}[0, T]$ reduces to the classical Wiener space $C_{0}[0, T]$ and $\left(e_{j}, a\right)_{C_{a, b}^{\prime}}=0$ for all $j=$ $1, \ldots, n$. Hence for all $\lambda \in \widetilde{\mathbb{C}}_{+}$,

$$
\left|\psi_{\vec{e}}(\lambda ; \vec{v})\right|=\left|\exp \left\{-\frac{1}{2 \lambda} \sum_{j=1}^{n} v_{j}^{2}\right\}\right|=\exp \left\{-\frac{\operatorname{Re}(\lambda)}{2|\lambda|^{2}} \sum_{j=1}^{n} v_{j}^{2}\right\} \leq 1 .
$$

Theorem 4.2. Let $F$ be given by the equation (3.4). Let $q_{0}$ be a positive real number. Suppose that the associated measure $\nu$ of $F$ satisfies condition (4.1) with $q$ replaced with $q_{0}$. Then for all real $q$ with $|q|>q_{0}$, the $L_{1} G F F T$ of $F$, $T_{q}^{(1)}(F)$ exists and is given by the formula

$$
T_{q}^{(1)}(F)(y)=\int_{\mathbb{R}^{n}} \exp \left\{i \sum_{j=1}^{n}\left(e_{j}, y\right)^{\sim} v_{j}\right\} \psi_{\vec{e}}(-i q ; \vec{v}) d \nu(\vec{v}),
$$

where $\psi_{\vec{e}}(-i q ; \vec{v})$ is given by the equation (3.8).

Proof. Let $\left\{\lambda_{l}\right\}_{l=1}^{\infty}$ be a sequence in $\mathbb{C}_{+}$such that $\lambda_{l} \rightarrow-i q$ through $\mathbb{C}_{+}$. Then $\psi_{\vec{e}}\left(\lambda_{l} ; \vec{v}\right)$ converges to $\psi_{\vec{e}}(-i q ; \vec{v})$. By Theorem 3.5 ,

$$
T_{\lambda_{l}}(F)(y)=\int_{\mathbb{R}^{n}} \exp \left\{i \sum_{j=1}^{n}\left(e_{j}, y\right)^{\sim}\right\} \psi_{\vec{e}}\left(\lambda_{l} ; \vec{v}\right) d \nu(\vec{v})
$$

exists for all $l \in \mathbb{N}$. Since $\left|\arg \left(\lambda_{l}^{-1 / 2}\right)\right|<\pi / 4$ for every $l \in \mathbb{N}$ and $\lambda_{l}^{-1 / 2}=$ $\operatorname{Re}\left(\lambda_{l}^{-1 / 2}\right)+i \operatorname{Im}\left(\lambda_{l}^{-1 / 2}\right) \rightarrow(-i q)^{-1 / 2}=1 / \sqrt{|2 q|}+i \operatorname{sign}(q) / \sqrt{|2 q|}$, we see that $\operatorname{Re}\left(\lambda_{l}^{-1 / 2}\right)>\left|\operatorname{Im}\left(\lambda_{l}^{-1 / 2}\right)\right|$ for every $l \in \mathbb{N}$ and there exists a sufficiently large 
$k \in \mathbb{N}$ such that $\left|\operatorname{Im}\left(\lambda_{l}^{-1 / 2}\right)\right|<1 / \sqrt{\left|q_{0}\right|}$ for every $l \geq k$. Thus for each $l \geq k$,

$$
\begin{aligned}
&\left|\psi_{\vec{e}}\left(\lambda_{l} ; \vec{v}\right)\right|=\mid \exp \{-\frac{1}{2}\left(\left[\operatorname{Re}\left(\lambda_{l}^{-1 / 2}\right)\right]^{2}-\left[\operatorname{Im}\left(\lambda_{l}^{-1 / 2}\right)\right]^{2}\right. \\
&\left.+i \operatorname{Re}\left(\lambda_{l}^{-1 / 2}\right) \operatorname{Im}\left(\lambda_{l}^{-1 / 2}\right)\right) \sum_{j=1}^{n} v_{j}^{2} \\
&\left.+i\left(\operatorname{Re}\left(\lambda_{l}^{-1 / 2}\right)+i \operatorname{Im}\left(\lambda_{l}^{-1 / 2}\right)\right) \sum_{j=1}^{n}\left(e_{j}, a\right)_{C_{a, b}^{\prime}} v_{j}\right\} \mid \\
&<\exp \left\{\frac{\|a\|_{C_{a, b}^{\prime}}}{\sqrt{\left|2 q_{0}\right|}} \sum_{j=1}^{n}\left|v_{j}\right|\right\}
\end{aligned}
$$

and so, by condition (4.1) with $q$ replaced with $q_{0}$,

$$
\begin{aligned}
\left|T_{\lambda_{l}}(F)(y)\right| & \leq \int_{\mathbb{R}^{n}}\left|\psi_{\vec{e}}\left(\lambda_{l} ; \vec{v}\right)\right| d|\nu|(\vec{v}) \\
& <\int_{\mathbb{R}^{n}} \exp \left\{\frac{\|a\|_{C_{a, b}^{\prime}}}{\sqrt{\left|2 q_{0}\right|}} \sum_{j=1}^{n}\left|v_{j}\right|\right\} d|\nu|(\vec{v})<+\infty .
\end{aligned}
$$

Also, by condition (4.1) with $q$ replaced with $q_{0}$, we have

$$
\begin{aligned}
& \left|\int_{\mathbb{R}^{n}} \exp \left\{i \sum_{j=1}^{n}\left(e_{j}, y\right)^{\sim} v_{j}\right\} \psi_{\vec{e}}(-i q ; \vec{v}) d \nu(\vec{v})\right| \\
\leq & \int_{\mathbb{R}^{n}}\left|\psi_{\vec{e}}(-i q ; \vec{v})\right| d|\nu|(\vec{v}) \\
< & \int_{\mathbb{R}^{n}} \exp \left\{\frac{\|a\|_{C_{a, b}^{\prime}}}{\sqrt{\left|2 q_{0}\right|}} \sum_{j=1}^{n}\left|v_{j}\right|\right\} d|\nu|(\vec{v})<+\infty .
\end{aligned}
$$

Hence by (3.5), (3.9) and the dominated convergence theorem, we have the equation (4.2).

The following corollary follows from equations (3.6) and (4.2).

Corollary 4.3. Let $F$ and $q_{0}$ be as in Theorem 4.2. Then for all real $q$ with $|q|>q_{0}$, the generalized analytic Feynman integral of $F, E^{\operatorname{anf}_{q}}[F]$ exists and is given by the formula

$$
E^{\operatorname{anf}_{q}}[F]=\int_{\mathbb{R}^{n}} \psi_{\vec{e}}(-i q ; \vec{v}) d \nu(\vec{v}),
$$

where $\psi_{\vec{e}}(-i q ; \vec{v})$ is given by the equation (3.8).

Theorem 4.4. Let $F$ and $q_{0}$ be as in Theorem 4.2. Then for all $p \in(1,2]$ and all real $q$ with $|q|>q_{0}$, the $L_{p}$ GFFT of $F, T_{q}^{(p)}(F)$ exists and is given by the right hand side of equation (4.2). 
Proof. It was shown in the proof of Theorem 3.5 that $T_{\lambda}(F)(y)$ given by equation (3.9) is an analytic function of $\lambda$ throughout $\mathbb{C}_{+}$. In view of the definition of the $L_{p}$ analytic GFFT, it suffices to show that for each $\rho>0$,

$$
\lim _{\lambda \rightarrow-i q} \int_{C_{a, b}[0, T]}\left|T_{\lambda}(F)(\rho y)-T_{q}^{(p)}(F)(\rho y)\right|^{p^{\prime}} d \mu(y)=0 .
$$

Given positive real number $q_{0}>0$, let $\Gamma_{q_{0}}=\left\{\lambda \in \tilde{\mathbb{C}}_{+}:\left|\operatorname{Im}\left(\lambda^{-1 / 2}\right)\right|<\right.$ $\left.\left(2 q_{0}\right)^{-1 / 2}\right\}$. Then for all real $q$ with $|q|>q_{0}$,

$$
(-i q)^{-1 / 2}=1 / \sqrt{2|q|}+i \operatorname{sign}(q) / \sqrt{2|q|} \in \Gamma_{q_{0}}
$$

and for all $\lambda \in \Gamma_{q_{0}}$,

$$
\left|\psi_{\vec{e}}(\lambda ; \vec{v})\right|<\exp \left\{\frac{\|a\|_{C_{a, b}^{\prime}}}{\sqrt{\left|2 q_{0}\right|}} \sum_{j=1}^{n}\left|v_{j}\right|\right\} .
$$

Fixing $p \in(1,2]$ and using inequalities (4.3) and (4.1), we obtain that for all $\rho>0$ and all $\lambda \in \Gamma_{q_{0}}$,

$$
\begin{aligned}
& \left|T_{\lambda}(F)(\rho y)-T_{q}^{(p)}(F)(\rho y)\right|^{p^{\prime}} \\
\leq & \left(\int_{\mathbb{R}^{n}}\left|\exp \left\{i \rho \sum_{j=1}^{n}\left(e_{j}, y\right)^{\sim}\right\}\right|\left\{\left|\psi_{\vec{e}}(\lambda ; \vec{v})\right|+\left|\psi_{\vec{e}}(-i q ; \vec{v})\right|\right\} d \nu(\vec{v})\right)^{p^{\prime}} \\
\leq & \left(2 \int_{\mathbb{R}^{n}} \exp \left\{\frac{\|a\|_{C_{a, b}^{\prime}}}{\sqrt{\left|2 q_{0}\right|}} \sum_{j=1}^{n}\left|v_{j}\right|\right\} d|\nu|(\vec{v})\right)^{p^{\prime}}<+\infty .
\end{aligned}
$$

Hence by the dominated convergence theorem, we see that for all $p \in(1,2]$ and all $\rho>0$,

$$
\begin{aligned}
& \lim _{\lambda \rightarrow-i q} \int_{C_{a, b}[0, T]}\left|T_{\lambda}(F)(\rho y)-T_{q}^{(p)}(F)(\rho y)\right|^{p^{\prime}} d \mu(y) \\
= & \int_{C_{a, b}[0, T]}\left|\int_{\mathbb{R}^{n}} \exp \left\{i \rho \sum_{j=1}^{n}\left(e_{j}, y\right)^{\sim}\right\} \lim _{\lambda \rightarrow-i q}\left\{\psi_{\vec{e}}(\lambda ; \vec{v})-\psi_{\vec{e}}(-i q ; \vec{v})\right\} d \nu(\vec{v})\right|^{p^{\prime}} d \mu(y) \\
= & 0
\end{aligned}
$$

which concludes the proof of Theorem 4.4.

\section{Sequential function space transforms}

In this section, we introduce two (non-analytic) sequential transforms of cylinder functionals discussed in Sections 3 and 4. We establish that the one is to identify the GFFT and the another transform acts like an inverse GFFT. 
For an orthonormal subset $\left\{e_{1}, \ldots, e_{n}\right\}$ of $C_{a, b}^{\prime}[0, T], \lambda \in \mathbb{C}_{+}$and $x \in$ $C_{a, b}[0, T]$, let

$$
\begin{aligned}
W_{\vec{e}}^{+}(\lambda ; x) \equiv \lambda^{n / 2} \exp \{ & \left(\frac{1-\lambda}{2}\right) \sum_{j=1}^{n}\left[\left(e_{j}, x\right)^{\sim}\right]^{2} \\
& \left.+\left(\lambda^{1 / 2}-1\right) \sum_{j=1}^{n}\left(e_{j}, a\right)_{C_{a, b}^{\prime}}\left(e_{j}, x\right)^{\sim}\right\}
\end{aligned}
$$

and

$$
\begin{aligned}
W_{\vec{e}}^{-}(\lambda ; x) \equiv & \lambda^{n / 2} \exp \left\{\left(\frac{1-\lambda}{2}\right) \sum_{j=1}^{n}\left[\left(e_{j}, x\right)^{\sim}\right]^{2}\right. \\
& \left.+\left((-\lambda)^{1 / 2}-1\right) \sum_{j=1}^{n}\left(e_{j}, a\right)_{C_{a, b}^{\prime}}\left(e_{j}, x\right)^{\sim}+\sum_{j=1}^{n}\left(e_{j}, a\right)_{C_{a, b}^{\prime}}^{2}\right\} .
\end{aligned}
$$

Definition 5.1. Let $F$ and $\left\{e_{1}, \ldots, e_{n}\right\}$ be related by the equation (3.2) and let $q$ be a nonzero real number. We define the sequential $\mathcal{P}$-transform of $F$ with parameter $q$ (if it exists) by

$$
\mathcal{P}_{\vec{e}, q}(F)(y)=\lim _{l \rightarrow \infty} \int_{C_{a, b}[0, T]} F(y+x) W_{\vec{e}}^{+}\left(\lambda_{l} ; x\right) d \mu(x),
$$

where $\left\{\lambda_{l}\right\}_{l=1}^{\infty}$ is any sequence in $\mathbb{C}_{+}$with $\lambda_{l} \rightarrow-i q$.

We also define the sequential $\mathcal{N}$-transform of $F$ with parameter $q$ (if it exists) by

$$
\mathcal{N}_{\vec{e}, q}(F)(y)=\lim _{l \rightarrow \infty} \int_{C_{a, b}[0, T]} F(y+x) W_{\vec{e}}^{-}\left(\lambda_{l} ; x\right) d \mu(x),
$$

where $\left\{\lambda_{l}\right\}_{l=1}^{\infty}$ is any sequence in $\mathbb{C}_{+}$with $\lambda_{l} \rightarrow-i q$.

Remark 5.2. Let $\left\{e_{1}, \ldots, e_{n}\right\}$ be an orthonormal subset $C_{a, b}^{\prime}[0, T]$. Then applying equations (2.1) and (2.2), we can see that for all $\lambda \in \mathbb{C}_{+}$,

$$
\int_{C_{a, b}[0, T]} W_{\vec{e}}^{+}(\lambda ; x) d \mu(x)=\int_{C_{a, b}[0, T]} W_{\vec{e}}^{-}(\lambda ; x) d \mu(x)=1 .
$$

In our next theorems we establish the existence of the sequential $\mathcal{P}$ and $\mathcal{N}$-transforms of functional $F$ given by equation (3.4).

Theorem 5.3. Let $F$ and $\left\{e_{1}, \ldots, e_{n}\right\}$ be related by the equation (3.4). Let $q_{0}$ be a positive real number. Suppose that the associated measure $\nu$ of $F$ satisfies condition (4.1) with $q$ replaced with $q_{0}$. Then for all real $q$ with $|q|>q_{0}$, the sequential $\mathcal{P}$-transform of $F, \mathcal{P}_{\vec{e}, q}(F)$ exists and is given by the right hand side of equation (4.2). 
Proof. Let $\left\{\lambda_{l}\right\}_{l=1}^{\infty}$ be any sequence in $\mathbb{C}_{+}$with $\lambda_{l} \rightarrow-i q$. By (3.4), (5.1), the Fubini theorem, (2.1) and (2.2), we have that for every $l \in \mathbb{N}$,

$$
\begin{aligned}
& \int_{C_{a, b}[0, T]} F(y+x) W_{\vec{e}}^{+}\left(\lambda_{l} ; x\right) d \mu(x) \\
= & \int_{\mathbb{R}^{n}} \exp \left\{i \sum_{j=1}^{n}\left(e_{j}, y\right)^{\sim} v_{j}\right\}\left(\frac{\lambda_{l}}{2 \pi}\right)^{n / 2} \int_{\mathbb{R}^{n}} \exp \left\{-\frac{\lambda_{l}}{2} \sum_{j=1}^{n} u_{j}^{2}\right. \\
& \left.\quad+\sum_{j=1}^{n}\left(\lambda_{l}^{1 / 2}\left(e_{j}, a\right)_{C_{a, b}^{\prime}}+i v_{j}\right) u_{j}-\frac{1}{2} \sum_{j=1}^{n}\left(e_{j}, a\right)_{C_{a, b}^{\prime}}^{2}\right\} d \vec{u} d \nu(\vec{v}) \\
= & \int_{\mathbb{R}^{n}} \exp \left\{i \sum_{j=1}^{n}\left(e_{j}, y\right)^{\sim} v_{j}-\frac{1}{2 \lambda_{l}} \sum_{j=1}^{n} v_{j}^{2}+i \lambda_{l}^{-1 / 2} \sum_{j=1}^{n}\left(e_{j}, a\right)_{C_{a, b}^{\prime}} v_{j}\right\} d \nu(\vec{v}) \\
= & \int_{\mathbb{R}^{n}} \exp \left\{i \sum_{j=1}^{n}\left(e_{j}, y\right)^{\sim} v_{j}\right\} \psi_{\vec{e}}\left(\lambda_{l} ; \vec{v}\right) d \nu(\vec{v}) .
\end{aligned}
$$

Applying (3.10), we see that the absolute value of the last expression of (5.3) is less than

$$
\exp \left\{\frac{\left|\lambda_{l}\right|^{2}\left(\operatorname{Im}\left(\lambda_{l}^{-1 / 2}\right)\right)^{2}}{2 \operatorname{Re}\left(\lambda_{l}\right)} \sum_{j=1}^{n}\left(e_{j}, a\right)_{C_{a, b}^{\prime}}^{2}\right\}\|\nu\|,
$$

and so the function space integral given by (5.3) exists for each $l \in \mathbb{N}$. Using equations (5.2), (5.3) and the dominated convergence theorem with the techniques similar to those used in the proof of Theorem 4.2, we have the desired result.

By similar methods, we obtain the following theorem.

Theorem 5.4. Let $F,\left\{e_{1}, \ldots, e_{n}\right\}$ and $q_{0}$ be as in Theorem 5.3. Then for all real $q$ with $|q|>q_{0}$, the sequential $\mathcal{N}$-transform of $F, \mathcal{N}_{\vec{e}, q}(F)$ exists and is given by the formula

$$
\begin{aligned}
\mathcal{N}_{\vec{e}, q}(F)(y)=\int_{\mathbb{R}^{n}} \exp \{ & i \sum_{j=1}^{n}\left(e_{j}, y\right)^{\sim} v_{j}-\frac{i}{2 q} \sum_{j=1}^{n} v_{j}^{2} \\
& \left.-i(i q)^{-1 / 2} \sum_{j=1}^{n}\left(e_{j}, a\right)_{C_{a, b}^{\prime}} v_{j}\right\} d \nu(\vec{v}) .
\end{aligned}
$$

Next we present concluding remarks.

(1) Let $F,\left\{e_{1}, \ldots, e_{n}\right\}$ and $q_{0}$ be as in Theorem 5.3. Then by Theorems 4.2 and 4.4, we have that for all real $q$ with $|q|>q_{0}$, the $\operatorname{GFFT} T_{q}^{(p)}(F)$ of $F$ is given by the right hand side of equation (4.2), and by Theorem 5.3, we have

$$
T_{q}^{(p)}(F)(y)=\mathcal{P}_{\vec{e}, q}(F)(y) .
$$



by

For nonzero real number $q$ with $|q|>q_{0}$, define a set function $\nu_{q}: \mathcal{B}\left(\mathbb{R}^{n}\right) \rightarrow \mathbb{C}$

$$
\nu_{q}(B)=\int_{\mathbb{R}^{n}} \psi_{\vec{e}}(-i q ; \vec{v}) d \nu(\vec{v}), \quad B \in \mathcal{B}\left(\mathbb{R}^{n}\right),
$$

where $\nu$ is the associated measure of $F$ and $\psi_{\vec{e}}(-i q ; \vec{v})$ is given by the equation (3.8). Then using condition (4.1) with $q$ replaced with $q_{0}$, we see that $\nu_{q}$ is a complex measure on $\mathcal{B}\left(\mathbb{R}^{n}\right)$ with finite total variation, and $T_{q}^{(p)}(F)$ and $\mathcal{P}_{\vec{e}, q}(F)$ can be expressed as

$$
T_{q}^{(p)}(F)(y)=\mathcal{P}_{\vec{e}, q}(F)(y)=\int_{\mathbb{R}^{n}} \exp \left\{i \sum_{j=1}^{n}\left(e_{j}, y\right)^{\sim} v_{j}\right\} d \nu_{q}(\vec{v})
$$

for all $p \in[1,2]$.

(2) For positive real number $q$ and complex measure $\nu_{q}$ discussed above, we assume

$$
\begin{aligned}
& \int_{\mathbb{R}^{n}} \exp \left\{\frac{\|a\|_{C_{a, b}^{\prime}}}{\sqrt{|2 q|}} \sum_{j=1}^{n}\left|v_{j}\right|\right\} d\left|\nu_{q}\right|(\vec{v}) \\
\leq & \int_{\mathbb{R}^{n}} \exp \left\{\frac{2\|a\|_{C_{a, b}^{\prime}}}{\sqrt{|2 q|}} \sum_{j=1}^{n}\left|v_{j}\right|\right\} d|\nu|(\vec{v})<+\infty .
\end{aligned}
$$

Then by equations (5.4) and (5.5), we have that for all $p \in[1,2]$ and all nonzero real number $q$,

$$
\begin{aligned}
\mathcal{N}_{\vec{e},-q}\left(T_{q}^{(p)}(F)\right)(y)= & \mathcal{N}_{\vec{e},-q}\left(\mathcal{P}_{\vec{e}, q}(F)\right)(y) \\
= & \int_{\mathbb{R}^{n}} \exp \left\{i \sum_{j=1}^{n}\left(e_{j}, y\right)^{\sim} v_{j}+\frac{i}{2 q} \sum_{j=1}^{n} v_{j}^{2}\right. \\
\left.\quad-i(-i q)^{-1 / 2} \sum_{j=1}^{n}\left(e_{j}, a\right)_{C_{a, b}^{\prime}} v_{j}\right\} d \nu_{q}(\vec{v}) & \\
= & \int_{\mathbb{R}^{n}} \exp \left\{i \sum_{j=1}^{n}\left(e_{j}, y\right)^{\sim} v_{j}+\frac{i}{2 q} \sum_{j=1}^{n} v_{j}^{2}\right. \\
= & \left.-i(-i q)^{-1 / 2} \sum_{j=1}^{n}\left(e_{j}, a\right)_{C_{a, b}^{\prime}} v_{j}\right\} \psi_{\vec{e}}(-i q ; \vec{v}) d \nu_{q}(\vec{v})
\end{aligned}
$$

By similar arguments we can see that $T_{-q}^{(p)}\left(\mathcal{N}_{\vec{e}, q}(F)\right)(y)=F(y)$. That is to say, the equation (5.7) tells us that sequential transform $\mathcal{N}_{\vec{e},-q}$ plays a prominent role as an inverse transform of GFFT $T_{q}^{(p)}$ for functionals $F$ given by equation $(3.4)$. 


\section{Examples}

In this section we present several interesting functionals to apply our results in previous sections.

Example 6.1. For given $\vec{m}=\left(m_{1}, \ldots, m_{n}\right) \in \mathbb{R}^{n}$ and $\overrightarrow{\sigma^{2}}=\left(\sigma_{1}^{2}, \ldots, \sigma_{n}^{2}\right) \in \mathbb{R}^{n}$ with $\sigma_{j}^{2}>0, j=1, \ldots, n$, let $\nu_{\vec{m}, \overrightarrow{\sigma^{2}}}$ be the Gaussian measure given by

$$
\nu_{\vec{m}, \overrightarrow{\sigma^{2}}}(B)=\left(\prod_{j=1}^{n} 2 \pi \sigma_{j}^{2}\right)^{-1 / 2} \int_{B} \exp \left\{-\sum_{j=1}^{n} \frac{\left(u_{j}-m_{j}\right)^{2}}{2 \sigma_{j}^{2}}\right\} d \vec{u}, \quad B \in \mathcal{B}\left(\mathbb{R}^{n}\right) .
$$

Then $\nu_{\vec{m}, \overrightarrow{\sigma^{2}}} \in \mathcal{M}\left(\mathbb{R}^{n}\right)$ and

$$
\widehat{\nu_{\vec{m}, \overrightarrow{\sigma^{2}}}}(\vec{u})=\exp \left\{-\frac{1}{2} \sum_{j=1}^{n} \sigma_{j}^{2} u_{j}^{2}+i \sum_{j=1}^{n} m_{j} u_{j}\right\} .
$$

Using the equation (6.1), the Fubini theorem and equation (2.2), we have that for all nonzero real number $q$,

$$
\begin{aligned}
& \int_{\mathbb{R}^{n}} \exp \left\{\frac{\|a\|_{C_{a, b}^{\prime}}}{\sqrt{|2 q|}} \sum_{j=1}^{n}\left|v_{j}\right|\right\} d\left|\nu_{\vec{m}, \overrightarrow{\sigma^{2}}}\right|(\vec{v}) \\
< & \prod_{j=1}^{n}\left[\exp \left\{\frac{\sigma_{j}^{2}\|a\|_{C_{a, b}^{\prime}}^{2}}{2|2 q|}-\frac{m_{j}\|a\|_{C_{a, b}^{\prime}}}{\sqrt{|2 q|}}\right\}+\exp \left\{\frac{\sigma_{j}^{2}\|a\|_{C_{a, b}^{\prime}}^{2}}{2|2 q|}+\frac{m_{j}\|a\|_{C_{a, b}^{\prime}}}{\sqrt{|2 q|}}\right\}\right]
\end{aligned}
$$

and the Gaussian measure $\nu_{\vec{m}, \overrightarrow{\sigma^{2}}}$ satisfies conditions (4.1) and (5.6) for all $q \in$ $\mathbb{R}-\{0\}$. Thus we can apply our results in previous sections to the functionals of the form

$$
\begin{aligned}
F_{1}(x) & =\widehat{\nu_{\vec{m}, \sigma^{2}}}\left(\left(e_{1}, x\right)^{\sim}, \ldots,\left(e_{n}, x\right)^{\sim}\right) \\
& =\exp \left\{-\frac{1}{2} \sum_{j=1}^{n} \sigma_{j}^{2}\left[\left(e_{j}, x\right)^{\sim}\right]^{2}+i \sum_{j=1}^{n} m_{j}\left(e_{j}, x\right)^{\sim}\right\},
\end{aligned}
$$

where $\left\{e_{1}, \ldots, e_{n}\right\}$ is an orthonormal set in $C_{a, b}^{\prime}[0, T]$.

By Theorems 4.2, 4.4, 5.3 and 5.4, for all $q \in \mathbb{R}-\{0\}$ and all $p \in[1,2]$, the $L_{p} \operatorname{GFFT} T_{q}^{(p)}\left(F_{1}\right)$, the sequential $\mathcal{P}$-transform $\mathcal{P}_{\vec{e}, q}\left(F_{1}\right)$ and the sequential $\mathcal{N}$-transform $\mathcal{N}_{\vec{e}, q}\left(F_{1}\right)$ exist and are given by

$$
\begin{aligned}
T_{q}^{(p)}\left(F_{1}\right)(y)= & \mathcal{P}_{\vec{e}, q}\left(F_{1}\right) \\
= & \left(\prod_{j=1}^{n} 2 \pi \sigma_{j}^{2}\right)^{-1 / 2} \int_{\mathbb{R}^{n}} \exp \left\{i \sum_{j=1}^{n}\left(e_{j}, y\right)^{\sim} v_{j}-\frac{i}{2 q} \sum_{j=1}^{n} v_{j}^{2}\right. \\
& \left.\quad+i(-i q)^{-1 / 2} \sum_{j=1}^{n}\left(e_{j}, a\right)_{C_{a, b}^{\prime}} v_{j}-\sum_{j=1}^{n} \frac{\left(v_{j}-m_{j}\right)^{2}}{2 \sigma_{j}^{2}}\right\} d \vec{v}
\end{aligned}
$$




$$
\begin{aligned}
=\left(\prod_{j=1}^{n} \frac{q}{q+i \sigma_{j}^{2}}\right)^{1 / 2} & \exp \left\{\sum _ { j = 1 } ^ { n } \frac { q \sigma _ { j } ^ { 2 } } { 2 ( q + i \sigma _ { j } ^ { 2 } ) } \left(i\left(e_{j}, y\right)^{\sim}\right.\right. \\
& \left.\left.+i(-i q)^{-1 / 2}\left(e_{j}, a\right)_{C_{a, b}^{\prime}}+\frac{m_{j}}{\sigma_{j}^{2}}\right)^{2}-\frac{m^{2}}{2 \sigma_{j}^{2}}\right\}
\end{aligned}
$$

and

$$
\begin{aligned}
\mathcal{N}_{\vec{e}, q}\left(F_{1}\right)=\left(\prod_{j=1}^{n} \frac{q}{q+i \sigma_{j}^{2}}\right)^{1 / 2} & \exp \left\{\sum _ { j = 1 } ^ { n } \frac { q \sigma _ { j } ^ { 2 } } { 2 ( q + i \sigma _ { j } ^ { 2 } ) } \left(i\left(e_{j}, y\right)^{\sim}\right.\right. \\
& \left.\left.-i(i q)^{-1 / 2}\left(e_{j}, a\right)_{C_{a, b}^{\prime}}+\frac{m_{j}}{\sigma_{j}^{2}}\right)^{2}-\frac{m^{2}}{2 \sigma_{j}^{2}}\right\},
\end{aligned}
$$

respectively. Furthermore,

$$
\begin{aligned}
\mathcal{N}_{\vec{e},-q}\left(T_{q}^{(p)}\left(F_{1}\right)\right)(y) & =\mathcal{N}_{\vec{e},-q}\left(\mathcal{P}_{\vec{e}, q}\left(F_{1}\right)\right)(y) \\
& =T_{-q}^{(p)}\left(\mathcal{N}_{\vec{e}, q}\left(F_{1}\right)\right)(y)=\mathcal{P}_{\vec{e},-q}\left(\mathcal{N}_{\vec{e}, q}\left(F_{1}\right)\right)(y)=F_{1}(y)
\end{aligned}
$$

for all $q \in \mathbb{R}-\{0\}$.

Example 6.2. Let $S: C_{a, b}^{\prime}[0, T] \rightarrow C_{a, b}^{\prime}[0, T]$ be the linear operator defined by $S w(t)=\int_{0}^{t} w(s) d b(s)$. Then the adjoint operator $S^{*}$ of $S$ is given by

$$
S^{*} w(t)=\int_{0}^{t}(w(T)-w(s)) d b(s) .
$$

It is easily shown that $S$ is injective. Let

$$
\gamma(t)=\sqrt{3} b^{-3 / 2}(T) b(t)
$$

Using an integration by parts formula, we see that $\left\{S^{*} \gamma\right\}$ is an orthonormal set in $C_{a, b}^{\prime}[0, T]$ and

$$
\frac{1}{\sqrt{3}} b^{3 / 2}(T)\left(S^{*} \gamma, x\right)^{\sim}=\left(S^{*} b, x\right)^{\sim}=\int_{0}^{T} x(t) d b(t) .
$$

In the case $n=1, e_{1}=S^{*} \gamma, \vec{m}=m_{1}=0$ and $\overrightarrow{\sigma^{2}}=\sigma_{1}^{2}=2 b^{3}(T) / 3$, the functional $F_{1}$ given by equation $(6.2)$ can be rewritten by

$$
F_{2}(x)=\exp \left\{-\left(\int_{0}^{T} x(t) d b(t)\right)^{2}\right\} .
$$

We give the transforms studied in this paper for functional $F_{2}$.

$$
\begin{aligned}
& T_{q}^{(p)}\left(F_{2}\right)(y) \\
= & \mathcal{P}_{\vec{S}^{*} \gamma, q}\left(F_{2}\right) \\
= & \left(\frac{3 q}{3 q+i 2 b^{3}(T)}\right)^{1 / 2} \exp \left\{\frac{3 q}{3 q+i 2 b^{3}(T)}\right.
\end{aligned}
$$




$$
\left.\times\left(i \int_{0}^{T} y(t) d b(t)+i(-i q)^{-1 / 2} \int_{0}^{T} a(t) d b(t)\right)^{2}\right\}
$$

and

$$
\begin{aligned}
& \mathcal{N}_{\vec{S}^{*} \gamma, q}\left(F_{2}\right)=\left(\frac{3 q}{3 q+i 2 b^{3}(T)}\right)^{1 / 2} \exp \left\{\frac{3 q}{3 q+i 2 b^{3}(T)}\right. \\
& \left.\times\left(i \int_{0}^{T} y(t) d b(t)-i(i q)^{-1 / 2} \int_{0}^{T} a(t) d b(t)\right)^{2}\right\} .
\end{aligned}
$$

Example 6.3. The functional

$$
F_{3}(x)=\exp \left\{i \int_{0}^{T} x(t) d b(s)\right\}
$$

also is a functional under our consideration because

$$
\begin{aligned}
F_{3}(x) & =\exp \left\{i\left(S^{*} b, x\right)^{\sim}\right\}=\exp \left\{\frac{i}{\sqrt{3}} b^{3 / 2}(T)\left(S^{*} \gamma, x\right)^{\sim}\right\} \\
& =\int_{\mathbb{R}} \exp \left\{i\left(S^{*} \gamma, x\right)^{\sim} v\right\} d \delta_{1}(v)=\widehat{\delta_{1}}\left(\left(S^{*} \gamma, x\right)^{\sim}\right),
\end{aligned}
$$

where $\gamma$ is given by equation $(6.3)$ and $\delta_{1}$ is the Dirac measure concentrated at $v=b^{3 / 2}(T) / \sqrt{3}$ in $\mathbb{R}$. Clearly, $\delta_{1}$ satisfies condition (4.1) and (5.6) for all $q \in \mathbb{R}-\{0\}$.

We finally present the two transforms of $F_{3}$.

$$
\begin{aligned}
T_{q}^{(p)}\left(F_{3}\right)(y) & =\mathcal{P}_{S^{*} \gamma, q}\left(F_{3}\right) \\
& =\exp \left\{i \int_{0}^{T} y(t) d b(t)-\frac{i}{6 q} b^{3}(T)+i(-i q)^{1 / 2} \int_{0}^{T} a(t) d b(t)\right\}
\end{aligned}
$$

and

$$
\mathcal{N}_{S^{*} \gamma, q}\left(F_{3}\right)=\exp \left\{i \int_{0}^{T} y(t) d b(t)-\frac{i}{6 q} b^{3}(T)-i(i q)^{1 / 2} \int_{0}^{T} a(t) d b(t)\right\} .
$$

Remark 6.4. The functionals given by equations (6.4) and (6.5) arise naturally in quantum mechanics.

\section{References}

[1] M. D. Brue, A functional transform for Feynman integrals similar to the Fourier transform, Thesis, University of Minnesota, Minneapolis, 1972.

[2] R. H. Cameron and D. A. Storvick, An $L_{2}$ analytic Fourier-Feynman transform, Michigan Math. J. 23 (1976), no. 1, 1-30.

[3] S. J. Chang, Conditional generalized Fourier-Feynman transform of functionals in a Fresnel type class, Commun. Korean Math. Soc. 26 (2011), no. 2, 273-289.

[4] S. J. Chang, J. G. Choi, and H. S. Chung, Generalized analytic Feynman integral via function space integral of bounded cylinder functionals, Bull. Korean Math. Soc. 48 (2011), no. 3, 475-489. 
[5] S. J. Chang, J. G. Choi, and D. Skoug, Integration by parts formulas involving generalized Fourier-Feynman transforms on function space, Trans. Amer. Math. Soc. 355 (2003), no. 7, 2925-2948.

[6] __ Generalized Fourier-Feynman transforms, convolution products, and first variations on function space, Rocky Mountain J. Math. 40 (2010), no. 3, 761-788.

[7] S. J. Chang and D. M. Chung, Conditional function space integrals with applications, Rocky Mountain J. Math. 26 (1996), no. 1, 37-62.

[8] S. J. Chang, H. S. Chung, and D. Skoug, Integral transforms of functionals in $L^{2}\left(C_{a, b}[0, T]\right)$, J. Fourier Anal. Appl. 15 (2009), no. 4, 441-462.

[9] S. J. Chang and D. Skoug, Generalized Fourier-Feynman transforms and a first variation on function space, Integral Transforms Spec. Funct. 14 (2003), no. 5, 375-393.

[10] T. Huffman, C. Park, and D. Skoug, Analytic Fourier-Feynman transforms and convolution, Trans. Amer. Math. Soc. 347 (1995), no. 2, 661-673.

[11] Convolutions and Fourier-Feynman transforms of functionals involving multiple integrals, Michigan Math. J. 43 (1996), no. 2, 247-261.

[12] _ Convolution and Fourier-Feynman transforms, Rocky Mountain J. Math. 27 (1997), no. 3, 827-841.

[13] G. W. Johnson and D. L. Skoug, Scale-invariant measurability in Wiener space, Pacific J. Math. 83 (1979), no. 1, 157-176.

[14] _ An $L_{p}$ analytic Fourier-Feynman transform, Michigan Math. J. 26 (1979), no. $1,103-127$.

[15] H. L. Royden, Real Analysis (Third edition), Macmillan, 1988.

[16] D. Skoug and D. Storvick, A survey of results involving transforms and convolutions in function space, Rocky Mountain J. Math. 34 (2004), no. 3, 1147-1175.

[17] J. Yeh, Singularity of Gaussian measures on function space induced by Brownian motion processes with non-stationary increments, Illinois J. Math. 15 (1971), 37-46.

[18] _ Stochastic Processes and the Wiener Integral, Marcel Dekker, Inc., New York, 1973.

JAe Gil Choi

Department of Mathematics

DANKOOK UNIVERSITY

Cheonan 330-714, Korea

E-mail address: jgchoi@dankook.ac.kr

Seung Jun Chang

Department of Mathematics

DANKOOK UNIVERSITY

Cheonan 330-714, Korea

E-mail address: sejchang@dankook.ac.kr 